GT2019-90179

\title{
DRAFT: A NEW DATA-DRIVEN TURBULENCE MODEL FRAMEWORK FOR UNSTEADY FLOWS APPLIED TO WALL-JET AND WALL-WAKE FLOWS
}

\author{
Chitrarth Lav; Jimmy Philip, Richard D. Sandberg \\ Department of Mechanical Engineering \\ University of Melbourne \\ Parkville, VIC 3010 \\ Email: clav@student.unimelb.edu.au
}

\begin{abstract}
The unsteady flow prediction for turbomachinery applications relies heavily on unsteady RANS (URANS). For flows that exhibit vortex shedding, such as the wall-jet/wake flows considered in this study, URANS is unable to predict the correct momentum mixing with sufficient accuracy. We suggest a novel framework to improve that prediction, whereby the deterministic scales associated with vortex shedding are resolved while the stochastic scales of pure turbulence are modelled. The framework first separates the stochastic from the deterministic length scales and then develops a bespoke turbulence closure for the stochastic scales using a data-driven machine-learning algorithm. The novelty of the method lies in the use of machine-learning to develop closures tailored to URANS calculations. For the walljet/wake flow, three different mass flow ratios $(0.86,1.07$ and 1.26) have been considered and a high-fidelity dataset of the idealised geometry is utilised for the sake of model development. This study serves as an a priori analysis, where the closures obtained from the machine-learning algorithm are evaluated before their implementation in URANS. The analysis looks at the impact of using all length scales versus the stochastic scales for closure development, and the impact of the extent of the spatial domain for developing the closure. It is found that a two-layer approach, using bespoke trained models for the near wall and the jet/wake regions, produce the best results. Finally, the generalisability of the developed closures is also evaluated by applying a given closure developed using a particular mass flow ratio to the other cases.
\end{abstract}

\section{NOMENCLATURE}

$u_{i} \quad$ Instantaneous $i^{t h}$ component of the velocity, $\bar{u}_{i}+u_{i}^{\prime}$

$\bar{u}_{i} \quad$ Time independent mean contribution of the velocity

$u_{i}^{\prime} \quad$ Instantaneous unsteadiness component of the velocity, $\widetilde{u}_{i}+u_{i}^{\prime \prime}$

$\widetilde{u}_{i} \quad$ Deterministic contribution of the velocity

$u_{i}^{\prime \prime} \quad$ Stochastic contribution of the velocity

$\begin{array}{ll}S_{i j} & \text { Mean strain rate tensor, } \frac{1}{2}\left(\frac{\partial \bar{u}_{i}}{\partial x_{j}}+\frac{\partial \bar{u}_{j}}{\partial x_{i}}\right) \\ \Omega_{i j} & \text { Mean rotation rate tensor, } \frac{1}{2}\left(\frac{\partial \bar{u}_{i}}{\partial x_{j}}-\frac{\partial \bar{u}_{j}}{\partial x_{i}}\right) \\ V_{i j}^{m} & \text { Tensor basis functions, } \forall m \in[1,2,3] \\ I_{m} & \text { Scalar invariant functions, } \forall m \in[1,2] \\ k & \text { Turbulence kinetic energy, TKE } \\ \omega & \text { Specific dissipation rate } \\ v_{t} & \text { Turbulent eddy viscosity } \\ \rho_{R S} & \text { Alignment } \\ \tau_{i j} & \text { Reynolds stress tensor, } \overline{u_{i}^{\prime} u_{j}^{\prime}} \\ a_{i j} & \text { Anisotropy tensor, } \frac{\tau_{i j}}{2 k}-\frac{\delta_{i j}}{3} \\ f & \text { Frequency } \\ f_{v s} & \text { Vortex shedding frequency } \\ \sigma & \text { Standard deviation of frequency distribution } \\ U_{f s} & \text { Freestream velocity in the main flow } \\ U_{b u l k} & \text { Bulk velocity in the slot } \\ s & \text { Height of the slot } \\ t & \text { Height of the lip separating the slot and main flow } \\ T_{f s}, T_{s l o t} & \text { Temperature in the freestream and slot respectively } \\ I_{f s}, I_{s l o t} & \text { Turbulence intensity in the freestream and slot respec- } \\ \text { Re } & \text { tively } \\ \text { St } & \text { Reynolds Number } \\ \text { LES } & \text { Strouhal Number } \\ \text { URANS } & \text { Large Eddy Simulation } \\ \text { GEP } & \text { Gene Expression Programming Reynolds-Averaged Navier Stokes } \\ \text { HiFi } & \text { High-fidelity } \\ \text { MFR } & \text { Mass Flow Ratio } \\ & \end{array}$

\section{INTRODUCTION}

The wall-jet and wall-wake flow is a configuration that has been studied experimentally by Kacker \& Whitelaw [1,2] and has direct application to turbomachinery flows such as high-pressure turbines [3]. This configuration has also been studied by Medic $\&$ Durbin [4] and Joo \& Durbin [5] numerically, through unsteady RANS (URANS), where overprediction of cooling efficiency was observed, attributed to the incorrect momentum mix- 
ing near the wall. The incorrect mixing is a result of the inability of URANS to predict sufficient unsteady coherent energy near the wall, i.e. not capturing the correct vortex shedding length scales. Effectively, the standard linear turbulence closure, i.e. the Boussinesq approximation, is accountable for the failure of URANS to predict the correct mixing. This issue was also observed by Ivanova \& Laskowski [6] and prediction improvements compared with the reference experiment [7] was possible only through scale resolving simulations such as Large Eddy Simulations (LES). However, using these simulations for industrial design is not ideal due to the high computational expense. Consequently, reliance on URANS for design continues and improving their predictive capability is of paramount importance. One way of doing that is by changing the turbulence closure. This has been investigated analytically before, in the form of Explicit Algebraic Reynolds Stress Models [8], with limited success. More recently however, a different approach of developing turbulence models has gained traction, that relies on machine-learning applied to high-fidelity ( $\mathrm{HiFi}$ ) datasets [9]. Of the different machinelearning efforts, the Gene Expression Programming (GEP) approach of Weatheritt \& Sandberg [10] is considered in this study. The GEP is an evolutionary algorithm that uses tensor regression to create a tensor model for a given tensor input. GEP has been chosen over other machine-learning approaches due to some key advantages:

1. The approach produces a tangible equation that can be implemented easily as a model into a solver [3].

2. Unlike methods such as neural-networks, GEP only requires HiFi datasets during the model development phase and not during the runtime of the RANS solver, which reduces the overall computational cost significantly [11].

This method for model development has been evaluated and applied in the context of steady RANS for various flow problems with promising improvements over the standard linear closure $[3,10,12]$. In contrast, it is used here to develop improved closures specifically for unsteady RANS approaches.

As mentioned before, one of the key problems in turbulence modelling is the Boussinesq approximation turbulence closure, which relates the modelled anistropy tensor $\left(a_{i j}^{\text {mod }}\right)$ with the mean strain rate tensor $\left(S_{i j}\right)$ through the eddy viscosity $\left(v_{t}\right)$ and turbulence kinetic energy $(k)$ :

$$
a_{i j}^{m o d}=-\frac{v_{t}}{k} S_{i j}=-\frac{S_{i j}}{\omega} .
$$

For the $k-\omega$ SST model [13], the above expression holds true. This closure is linear since the mean strain rate tensor is directly proportional to the modelled anisotropy tensor. The GEP algorithm can be used instead to develop a non-linear turbulence closure, using additional terms, called tensor basis functions and scalar invariants [14]

$$
\begin{aligned}
V_{i j}^{1} & =\frac{S_{i j}}{\omega}, \quad I_{1}=S_{m n} S_{n m}, \quad I_{2}=\Omega_{m n} \Omega_{n m}, \\
V_{i j}^{2} & =\frac{S_{i k} \Omega_{k j}-\Omega_{i k} S_{k j}}{\omega}, \\
V_{i j}^{3} & =\frac{S_{i k} S_{k j}-\frac{1}{3} \delta_{i j} S_{m n} S_{n m}}{\omega}
\end{aligned}
$$

i.e. $a_{i j}^{\text {mod }}=f\left(V_{i j}^{1}, V_{i j}^{2}, V_{i j}^{3}, I_{1}, I_{2}\right)$. A good way to assess the modelled anisotropy's effectiveness is by computing the alignment [15], which represents the normalised inner product of the modelled anisotropy with the reference anisotropy

$$
\rho_{R S}=\frac{a_{i j}^{r e f} a_{j i}^{\text {mod }}}{\sqrt{a_{m n}^{r e f} a_{n m}^{r e f} a_{p q}^{\text {mod }} a_{q p}^{\text {mod }}}} .
$$

Here, the reference anisotropy is extracted from the HiFi dataset as

$$
a_{i j}^{r e f}=\frac{\tau_{i j}}{2 k}-\frac{\delta_{i j}}{3} .
$$

A completely valid model would exhibit perfect alignment with a value of 1.0, while models that are orthogonal would have a value of 0.0 and models with a value of -1.0 are oppositely aligned. This parameter will be used extensively in this study to evaluate the different models obtained via machine-learning.

In this paper, the machine-learning process is applied to develop non-linear turbulence closures for improving prediction of the momentum mixing in wall-jet/wake flow through a new framework for unsteady flows. Crucially, the closures are only considering the stochastic part of the unsteady flow, extracted from the high-fidelity data. Three different mass flow ratios have been considered, for which the turbulence closures have been developed through LES datasets. The paper is organised into three sections: methodology, the reference dataset and the results. The first part will detail the new framework for unsteady flows. The second part will provide details of the domain considered for the study and the application of the new framework to extract relevant information from the HiFi data. Finally, the third part will explore the impact of the framework on the improvements using the developed turbulence closures.

\section{METHODOLOGY}

The framework considered in this study is adapted from Lav et al. [16], which can be summarised through the blue arrows in the schematic in Fig. 1. The original framework developed for steady flows is also shown using the red arrows [10]. For steady flows, the machine-learning process or "training" involved developing a turbulence closure using all the length scales from the HiFi dataset i.e. using the full anisotropy tensor $\left(a_{i j}^{r e f}\right)$. The resulting model $\left(a_{i j}^{x f}\right)$ would then be added to a modified turbulence closure for implementation in RANS

$$
\tau_{i j}^{R A N S}=\frac{2}{3} k \delta_{i j}-2 v_{t} S_{i j}+2 k a_{i j}^{x f}
$$

This methodology was applied to the wall-jet/wake flow in Sandberg et al [3], with limited improvement. Representing this case in an unsteady sense, however, would require a modification to the turbulence closure development, due to the existence of the vortex shedding length scales. In an unsteady calculation such as URANS, the vortex shedding scales are captured while the remaining scales are modelled with the turbulence closure. A closure developed using all the length scales, as in the original 


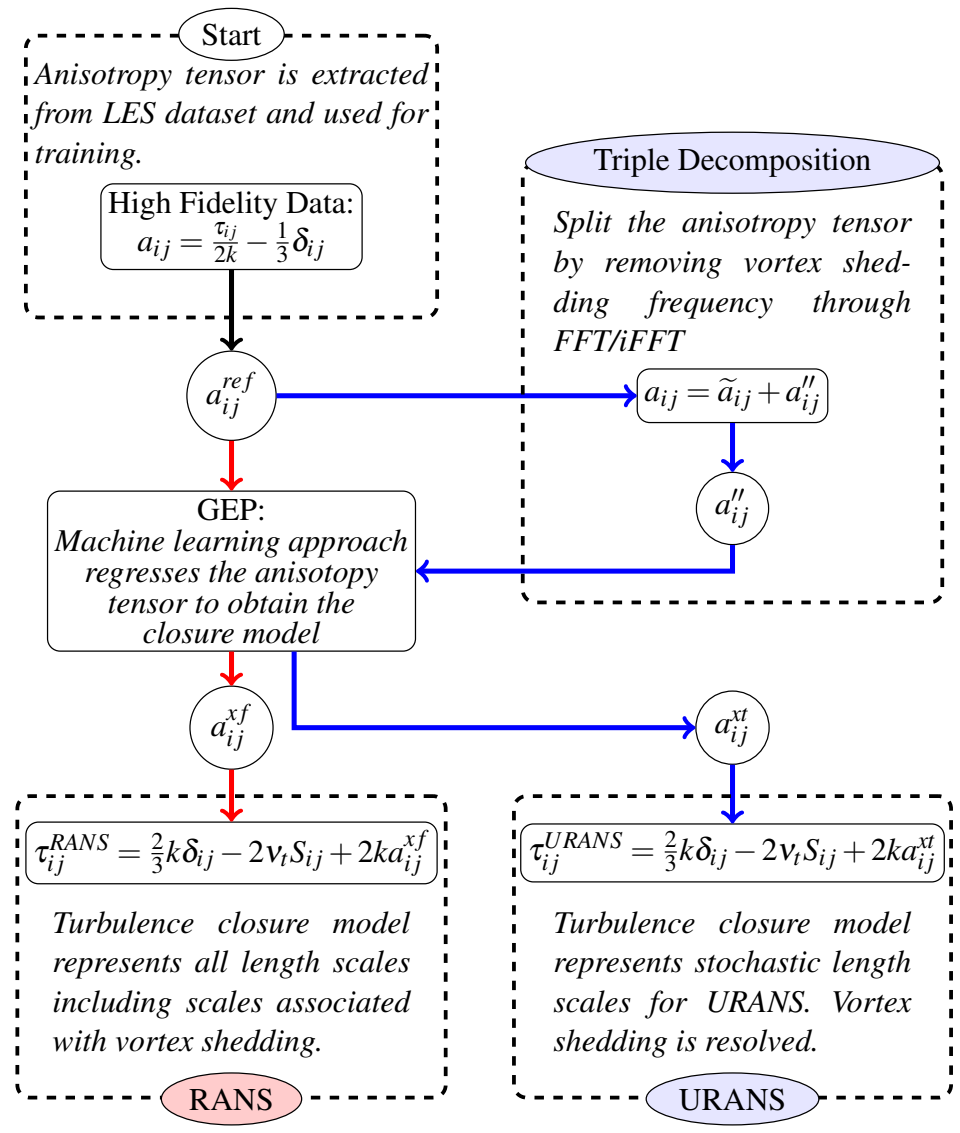

FIGURE 1. Schematic of the proposed framework in blue arrows, adapted from Lav et al. [16].

framework, would result in accounting for the shedding scales twice: one, using the developed turbulence closure and two, by being captured by URANS. Also, vortex shedding signifies presence of coherent length scales, which are not part of the turbulence, so turbulence closure development should not include contributions from these scales. Thus, the modification of the original framework for unsteady flows is to develop a closure that is devoid of the vortex shedding length scales. This can be achieved by considering a triple decomposition split [17]

$$
\begin{aligned}
u(\mathbf{x}, t) & =\bar{u}(\mathbf{x})+u^{\prime}(\mathbf{x}, t), \\
& =\bar{u}(\mathbf{x})+\widetilde{u}(\mathbf{x}, t)+u^{\prime \prime}(\mathbf{x}, t) .
\end{aligned}
$$

The length scales associated with the vortex shedding, also referred to as deterministic scales, are contained within $\widetilde{u}$ while the remaining unsteady scales, referred to as stochastic, contained within $u^{\prime \prime}$. Classed together, these terms give rise to the Reynolds decomposition. The result of the triple decomposition of the Reynolds stress tensor (as well as the TKE and the anisotropy) shows that the stress is a sum of the deterministic and stochastic contributions:

$$
\begin{aligned}
\tau_{i j} & =\overline{u_{i}^{\prime} u_{j}^{\prime}}, \\
& =\overline{\left(\widetilde{u}_{i}+u_{i}^{\prime \prime}\right)\left(\widetilde{u}_{j}+u_{j}^{\prime \prime}\right)}, \\
& =\overline{\widetilde{u}_{i} \widetilde{u}_{j}}+\overline{u_{i}^{\prime \prime} u_{j}^{\prime \prime}}, \\
& =\widetilde{\tau}_{i j}+\tau_{i j}^{\prime \prime} .
\end{aligned}
$$

This split is performed on the anisotropy tensor from the HiFi dataset and only the stochastic component $\left(a_{i j}^{\prime \prime}\right)$ is used to develop the turbulence closure as opposed to the full anisotropy tensor in the original framework. The resulting closure model $\left(a_{i j}^{x t}\right)$ is then added to a similarly modified turbulence closure

$$
\tau_{i j}^{U R A N S}=\frac{2}{3} k \delta_{i j}-2 v_{t} S_{i j}+2 k a_{i j}^{x t}
$$

The developed closure then accounts for the stochastic length scales while the deterministic scales are resolved. Equations (5) and (8) show the modelled Reynolds stress tensor, from which the modelled anisotropy $\left(a_{i j}^{\text {mod }}\right)$ can be obtained by moving the Kronecker delta term to the left side of the equation and dividing the expressions with $2 k$ to non-dimensionalise the equations. The eddy viscosity terms in the equations get transformed to the first tensor basis function $V_{i j}^{1}$ through Eqn. (1). Thus, the modelled anisotropy from each method is:

$$
\begin{gathered}
\left.a_{i j}^{\text {mod }}\right|_{R A N S}=\frac{\tau_{i j}^{R A N S}}{2 k}-\frac{1}{3} \delta_{i j}=-V_{i j}^{1}+a_{i j}^{x f}, \\
\left.a_{i j}^{\text {mod }}\right|_{U R A N S}=\frac{\tau_{i j}^{U R A N S}}{2 k}-\frac{1}{3} \delta_{i j}=-V_{i j}^{1}+a_{i j}^{x t} .
\end{gathered}
$$

\section{REFERENCE DATASET}

The dataset for the machine-learning or "training" process was obtained by conducting three high-resolution LES using the WALE model [18] with the in-house solver HiPSTAR [19]. The LESs were benchmarked with the experimental data from Kacker $\&$ Whitelaw [2]. The details of the domain and grid resolution are provided in Sandberg et al [3], with the domain schematic shown in Fig. 2 and the flow conditions in Tab. 1. The schematic

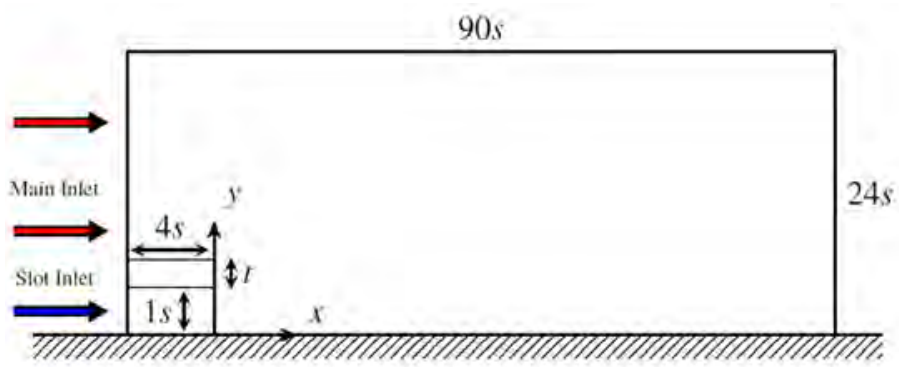

FIGURE 2. Schematic of the domain [3].

shows a co-flow separated by a lip of finite thickness $t$. The slot ejects fluid forming a film along the lower wall with the velocity of ejection defined in relation to the freestream velocity in the main flow. Three Mass Flow Ratios (MFRs) are considered in this study with values specified in Tab. 1. MFR is defined as the ratio of the bulk velocity of the slot flow $\left(U_{b u l k}\right)$ to the freestream velocity in the main flow $\left(U_{f s}\right)$. Given the MFRs, the flow can be classed as a wall-jet for MFR $>1.0$ and wallwake for MFR $<1.0$ [2]. The length and velocity scales are nondimensionalised with $s$ and $U_{f s}$ respectively. Figure 3 shows an instantaneous snapshot of the Q-criterion [20], coloured by the spanwise vorticity (along $z$ direction) for the MFR $=1.26$ case to illustrate the different length scales in the flow. 


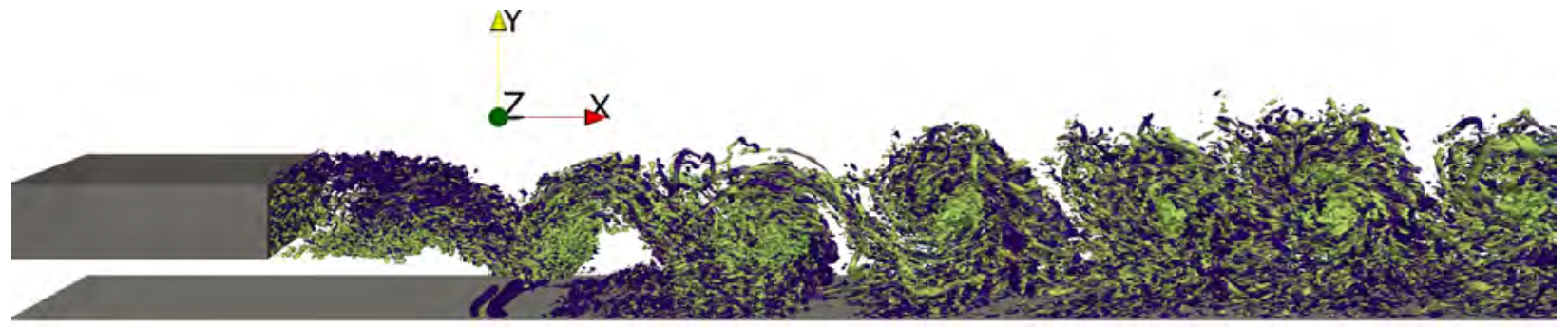

FIGURE 3. Instantaneous snapshot of Q-criterion, coloured by spanwise vorticity for MFR = 1.26.

TABLE 1. Simulation Parameters

\begin{tabular}{c|c}
\hline \hline Parameters & Values \\
\hline \hline$U_{\text {bulk }}$ & $16.5,20.5,24.2 \mathrm{~m} / \mathrm{s}$ \\
\hline$U_{f s}$ & $19.2 \mathrm{~m} / \mathrm{s}$ \\
\hline$I_{\text {slot }}$ & $5 \%$ \\
\hline$I_{\text {main }}$ & $0.5 \%$ \\
\hline$l_{t}$ & $10 \%$ of slot height \\
\hline MFR & $0.86,1.07,1.26$ \\
\hline$t / s$ & 1.14 \\
\hline$R e_{\text {slot }}$ & 12,000 \\
\hline$T_{\text {slot }}$ & $273 \mathrm{~K}$ \\
\hline$T_{f s}$ & $323 \mathrm{~K}$ \\
\hline \hline
\end{tabular}

With the given dataset, the first part of the framework is considered, i.e., separating the deterministic from the stochastic unsteadiness. The deterministic unsteadiness is visible as vortex shedding in Fig. 3. The range of deterministic time scales (or equivalent length scales) can be evaluated by looking at the turbulence spectra for the three MFRs, shown in Fig 4.

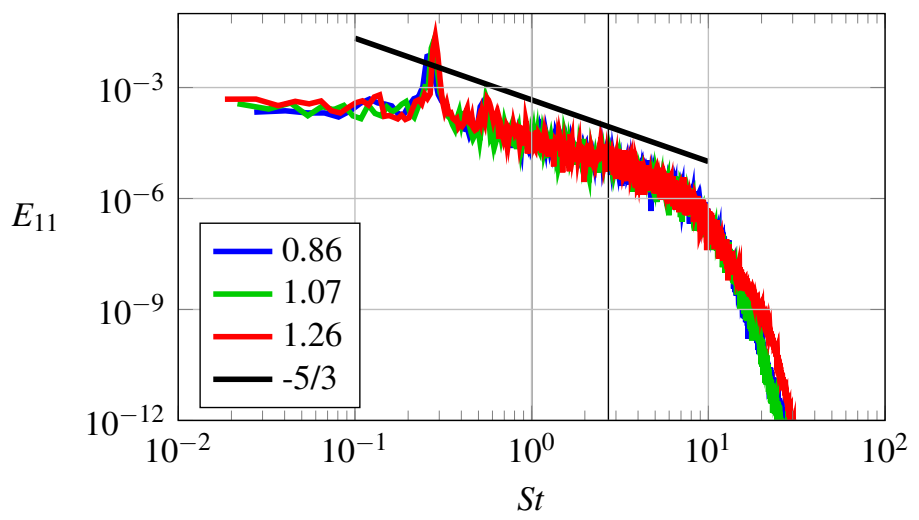

FIGURE 4. Streamwise velocity $\left(u_{i}\right)$ turbulent energy spectra for the 3 mass flow ratios at $(x, y)=(6.0,0.5)$ units. $-5 / 3$ line has also been added for reference.

This figure shows the presence of a dominant peak associated with the vortex shedding. The frequencies associated with the shedding $\left(f_{v s}\right)$ can be calculated from the Strouhal number (St) by considering the MFR and the lip thickness as the velocity and length scale, resulting in 0.2035 (MFR = 0.86), 0.2645 (MFR $=1.07)$ and $0.3153(\mathrm{MFR}=1.26)$ as the frequencies. While Joo $\&$ Durbin [5] argue that the deterministic and stochastic scales are not independent, the separation of the deterministic scales from the broadband spectrum of turbulence is possible here due to the deterministic energy spread in a narrow band of frequencies, evident from the sharp peak in the spectra in Fig. 4.

Based on the obtained frequencies, 400 time samples at an interval of 400 iterations with a timestep of 0.0006 time units were collected. This resulted in the time series containing $\sim 20$ $(\mathrm{MFR}=0.86), 25(\mathrm{MFR}=1.07)$ and $30(\mathrm{MFR}=1.26)$ vortex shedding cycles, which is found sufficiently long to represent the deterministic unsteadiness. These time series were then subjected to Fast-Fourier Transforms (FFTs) where the Fourier coefficients of the velocities were multiplied with a Gaussian distribution $(H(f))$, centered at $f_{v s}$. Eight frequencies on either side of $f_{v s}$ made up $6 \sigma$, where $\sigma$ represents the standard deviation

$$
H(f)=\exp \left(\frac{-\left(f-f_{v s}\right)^{2}}{2 \sigma^{2}}\right)
$$

An inverse Fourier transform of the resulting signal then provides a representation of the deterministic unsteadiness $\left(\widetilde{u}_{i}\right)$. The Gaussian distribution for filtering was chosen for two reasons: one, to prevent ringing in the data due to the Gibb's phenomenon and two, to better reflect the deterministic scales' distribution, as also seen from the shape of the peak in the energy spectra (Fig. 4). Subsequent time and span averaging of the data results in the deterministic Reynolds stress $\left(\widetilde{\widetilde{u}}_{i} \widetilde{u}_{j}\right)$, from which the stochastic contribution is obtained through Eqn. (7). An example of this split is shown in Fig. 5, for MFR $=1.26$ at $x=10.0$ units downstream. It is evident from this figure that the deterministic contribution is comparable to the stochastic part of the Reynolds stress, which would explain why developing turbulence closures using the total/full anisotropy showed limited improvements in Sandberg et al [3]. The stochastic anisotropy $\left(a_{i j}^{\prime \prime}\right)$ is thus used to develop the turbulence closure.

\section{RESULTS}

Turbulence closure development with GEP relies on providing the anisotropy tensor for a given spatial extent. In this section, the quality of the developed closures are evaluated for different spatial extents, or, training regions. To this end, the following section is divided further into three parts: stochastic versus full anisotropy for turbulence closure development, assessing the 

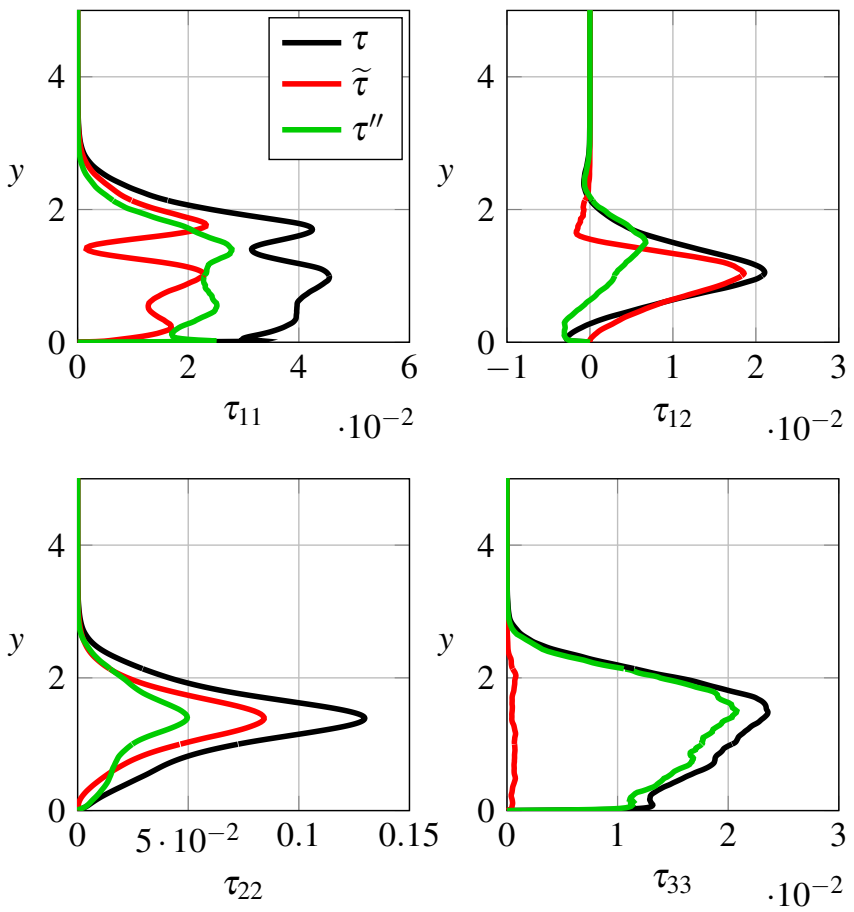

FIGURE 5. Total $(\tau)$, deterministic $(\widetilde{\tau})$ and stochastic $\left(\tau^{\prime \prime}\right)$ Reynolds stresses for MFR $=1.26$ at location $x=10.0$.

impact of the training region on the obtained turbulence closure and the development of zonal turbulence closures.

\section{Effect of stochastic versus full anisotropy}

As a start, the training region considered for developing the closures was chosen to be 5.0-30.0 and 0.0-3.0 units in the $x$ and $y$ directions, respectively. The limits in the $x$ direction are chosen such that the locations are downstream of the mean recirculation region and include a wide range of length scales. The limits in the $y$ direction are chosen to include the regions of large variations in the strain rates. The effect of the training region on the resulting models will be revisited in the later sections.

The need for developing the non-linear closure using the new framework is demonstrated from the alignment contours (Eqn. (3)) in Fig. 6. As mentioned earlier, a model's validity is confirmed from the positive values of alignment, visually from the amount of redness in the contour. The terms in the bracket of $\rho_{R S}$ represent the quantity $a_{i j}^{\text {mod }}$ in Eqn. (3). Training with the full anisotropy tensor results in a model denoted by $a_{i j}^{x f}$ while the stochastic anisotropy model is denoted by $a_{i j}^{x t}$. Since these models are added on as extra anisotropies to the Boussinesq approximation (see Fig. 1), the effective anisotropy closures using the full anisotropy is denoted by $a_{i j}^{\text {mod }}=a_{i j}^{x f}-V_{i j}^{1}$ (Eqn. (9)) and using the stochastic anisotropy is denoted by $a_{i j}^{\text {mod }}=a_{i j}^{x t}-V_{i j}^{1}$ (Eqn. (10)). The standard closure (Boussinesq approximation) shows extremely poor values of alignment everywhere except for a thin layer away from the wall, which serves as an interface between the shear layer of the boundary layer and the free shear layer of the jet/wake. The closure developed from the full anisotropy $\left(a_{i j}^{x f}\right)$ tensor shows regions of significantly improved alignment. However, the region near the wall suffers from near orthonormal values. This is rectified to an extent when stochastic anisotropy $\left(a_{i j}^{x t}\right)$ is used to develop the closure. The improvement here is attributed to the removal of the deterministic scales,
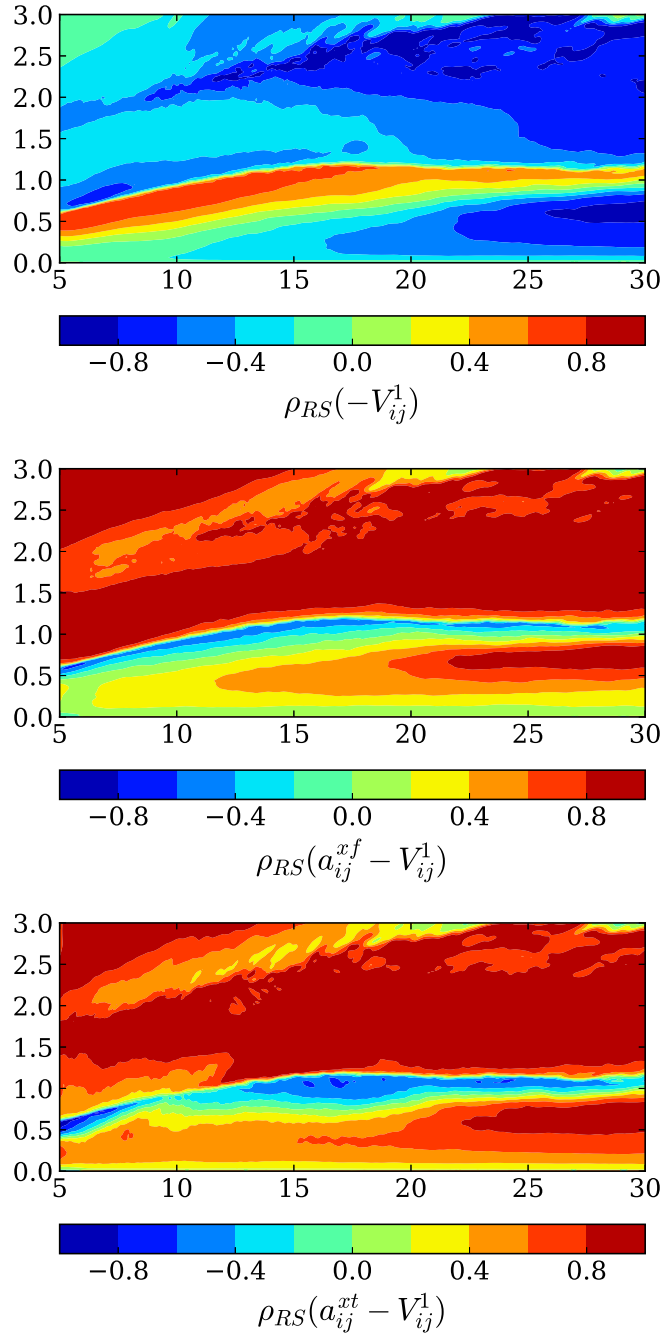

FIGURE 6. Alignment contours for (top) Boussinesq (middle) full anisotropy $\left(a_{i j}^{x f}\right)$ (bottom) stochastic anisotropy closures $\left(a_{i j}^{x t}\right)$. MFR $=$ 1.26 .

which are dominant further upstream and in the near wall region, as seen in Fig. 5. These observations remain consistent for the other two mass flow ratios as well, which are not shown for brevity. Thus, model development as part of the new framework incorporating the stochastic anisotropy is advantageous and is used throughout the rest of the results section.

Figure 6, however, also shows that there are regions where alignments are not improved by the closures developed through either approach. This will be addressed in the following sections.

\section{Impact of Training Region}

The choice of the training region has an influence on the quality of the developed closure. For the wall-jet/wake flow, it has shown to be of considerable impact [3]. In this study, a similar treatment is extended when training with the stochastic anisotropy. Models presented here are applied everywhere in the domain. Since the training from 0.0-3.0 units in $y$ failed to significantly improve the alignment in the near wall region, a region from 0.0-1.0 units in $y$ with 5.0-30.0 units in $x$ was chosen.

Figure 7 shows the contours of alignment for a closure developed on MFR = 1.26 that has been applied to all the MFRs. The colourbar label represents the reference and models, i.e. 

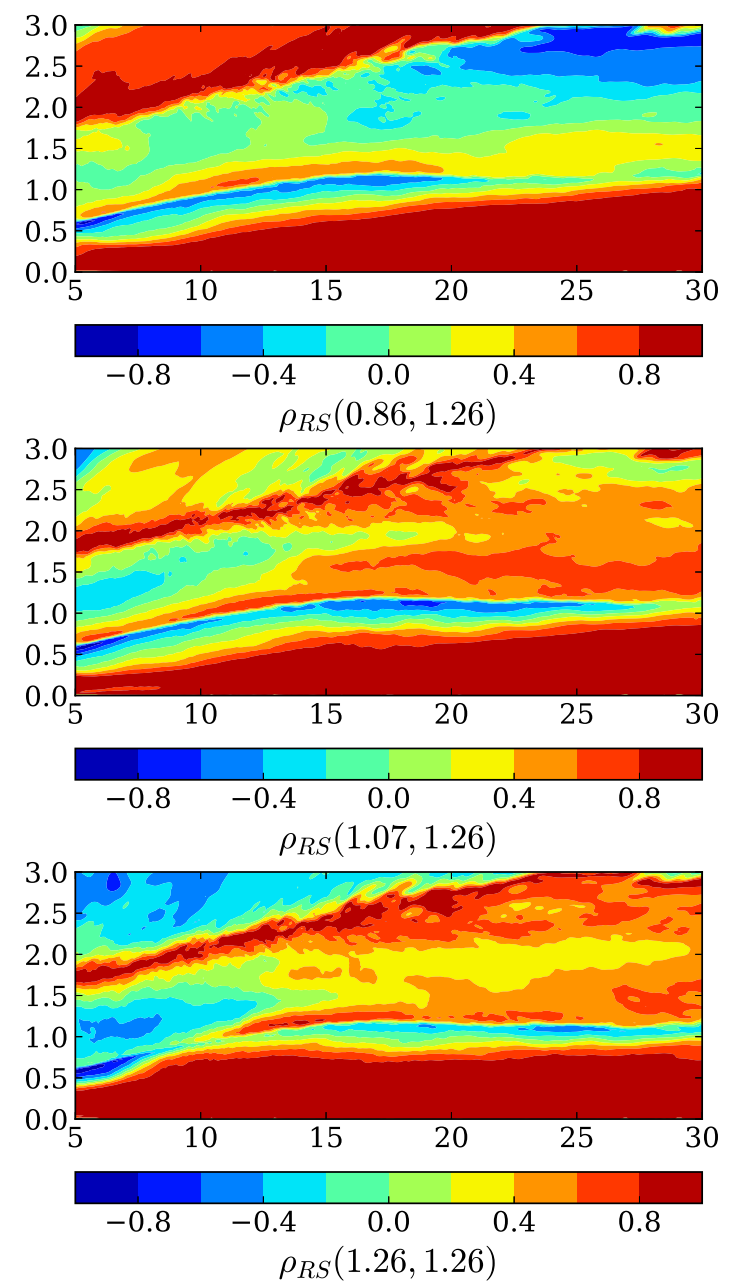

FIGURE 7. Alignment contours for training region 5.0-30.0 units and 0.0-1.0 in the $x$ and $y$ directions, applied from 5.0-30.0 units and 0.0-3.0 units in the $x$ and $y$ directions. Training on MFR $=1.26$ and applied on $\mathrm{MFR}=0.86,1.07 \& 1.26$.

$\rho_{R S}(0.86,1.26)$ refers to the alignment between reference from $\mathrm{MFR}=0.86$ and model from $\mathrm{MFR}=1.26$. These contours give an idea of whether the developed model is applicable for other MFRs. As can been seen, the alignment in the near wall has been significantly improved, when compared with the original training region (Fig. 6) and the model seems applicable to the remaining MFRs. These trends were consistent on the other permutations of alignment with the remaining MFRs. However, away from the wall, where $y>1.0$, the alignment values are worse than with the original training region. This suggests the developed closure should only be applied in regions where $y<1.0$.

To improve the alignment values for $y>1.0$, another training region is considered, spanning from 1.0-5.0 units in $y$. The upper limit is changed from 3.0 to 5.0 to account for a band of low alignment values that moves out of the plot in Fig. 6. The lower limit is changed to 1.0 from 0.0 to obtain a closure that is tuned for the region away from the wall. Figure 8 shows the alignment values for the training region of 5.0-30.0 units in $x$ and 1.0-5.0 in $y$ directions, applied from 5.0-30.0 units in $x$ and 0.0-5.0 units in $y$ directions. As before, the model developed on MFR $=1.26$ is applied to all the other MFRs as well to test the generalisability. Clearly, alignment away from the wall has improved dramatically when compared with Fig. 7, at the cost of
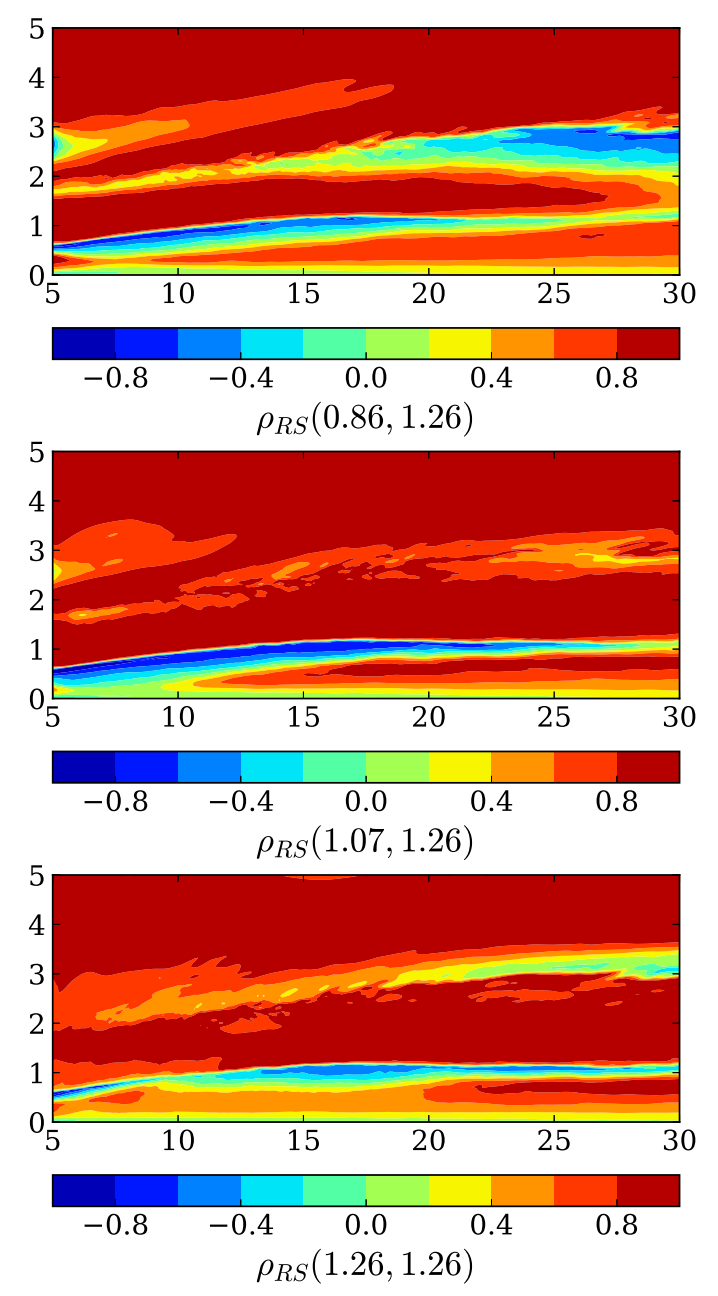

FIGURE 8. Alignment contours for training region 5.0-30.0 units and 1.0-5.0 in the $x$ and $y$ directions, applied from 5.0-30.0 units and 0.0-5.0 in the $x$ and $y$ directions. Training performed on MFR $=1.26$ and applied on MFR $=0.86,1.07 \& 1.26$.

the alignment near the wall.

The analysis with the different training regions in this section shows that one trained closure applied everywhere is not capable of depicting the complete flow physics. The next section addresses this issue by considering different closures for different regions.

\section{Zonal Turbulence Closures}

Following the findings in the previous section, closures are developed on specific flow physics. A simplified description of the mean flow physics would involve presence of a boundary layer along the lower wall. This layer merges into a wake (MFR $<1.0$ ) or a jet (MFR $>1.0$ ) away from the wall, produced by the lip separating the slot flow from the main flow. Thus, a simple start in the training process would consider two training regions: 0.0-1.0 units and 1.0-5.0 units in the $y$ direction with 5.0-30.0 in the $x$ direction. The alignment contours for the two closures stiched together are shown in Fig. 9. The figure shows that even though there is a better overall agreement in the alignment when compared with one turbulence closure, the alignment values in the interface between the two zones shows completely different values, which in an a posteriori analysis would likely produce 

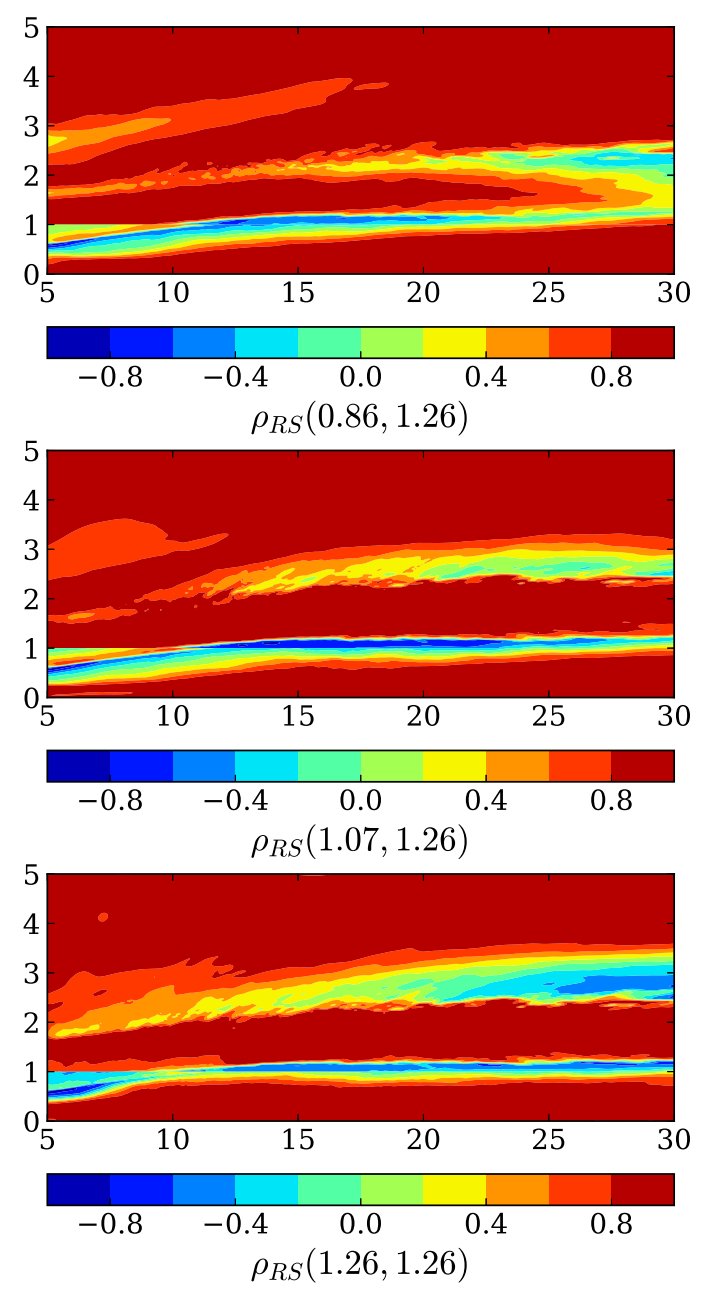

FIGURE 9. Alignment contours for training region 5.0-30.0 units in $x$ with 0.0-1.0 and 1.0-5.0 in $y$ directions, applied from 5.0-30.0 units in $x$ with $0.0-1.0$ and 1.0-5.0 in $y$ directions. Training performed on MFR $=1.26$ and applied on MFR $=0.86,1.07 \& 1.26$.

numerical oscillations in the mean flow profiles. To better represent the stitching between the two zones, we propose a zone demarcation that relies on splitting the boundary layer and the jet/wake. This can be performed by considering a curve in the $x-y$ plane where $\partial \bar{u} / \partial y=0$ the first time on increasing $y$ for a given $x$ location. The datapoints for all three MFRs are plotted in Fig. 10. It can be seen that the demarcation lines across the different MFR's are similiar, which allows specification of just one curve (also shown in Fig. 10), approximated with a cubic fit:

$$
y=0.00011354 x^{3}-0.0079756 x^{2}+0.18042 x-0.1388
$$

The training process is performed by considering two regions, one below the curve in Eqn. (12) and one above the curve. The resulting models are identified by superscripts $B$ for boundary layer region (under the curve) and $J$ for the jet/wake region (over the curve).

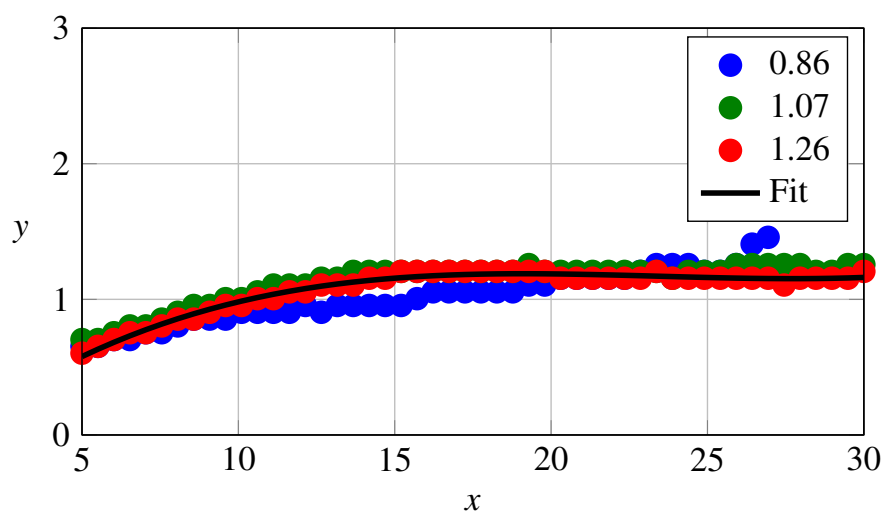

FIGURE 10. Boundary layer and jet zone separation for the 3 MFRs including a cubic curve fit.

\section{MFR = 0.86:}

$$
\begin{aligned}
a_{i j}^{B} & =\left(I_{1}\left(2-I_{2}\right)\left(3 I_{1}-2 I_{2}+1\right)-0.619\right) V_{i j}^{1} \\
& -\left(I_{1}+5.786\right) V_{i j}^{2} \\
& -\left(2 I_{1}\left(I_{1}-2\right)\left(4 I_{1}-2\right)+2 I_{1}+I_{2}-5.176\right) V_{i j}^{3}, \\
a_{i j}^{J} & =\left(3 I_{1}-I_{1}^{2}-1.0\right) V_{i j}^{1} \\
& -\left(I_{1}+I_{2}\left(I_{1}+1.88\right)\left(2 I_{1}-I_{2}\right)+I_{2}\right) V_{i j}^{2} \\
& +\left(1.107 I_{1}\left(I_{2}-I_{2}\right)+5.37 I_{2}-4.786 I_{1}+5.167\right) V_{i j}^{3} .
\end{aligned}
$$

MFR = 1.07:

$$
\begin{aligned}
a_{i j}^{B} & =\left(2-I_{2}\right)\left(I_{2}+\left(I_{1}-I_{2}\right)\left(I_{2}+0.85\right)-0.15\right) V_{i j}^{1} \\
& -\left(2 I_{2}-4.85\right)\left(2 I_{2}-1\right) V_{i j}^{2} \\
& +\left(5 I_{1}+3.547\right) V_{i j}^{3}, \\
a_{i j}^{J} & =\left(3.718 I_{1}-1.718\right)\left(I_{2}+0.58\right) V_{i j}^{1} \\
& +\left(-0.42 I_{1}+1.16 I_{2}+0.58\right) V_{i j}^{2} \\
& +\left(-5 I_{1}+5 I_{2}+4.518\right) V_{i j}^{3} .
\end{aligned}
$$

\section{MFR = 1.26:}

$$
\begin{aligned}
a_{i j}^{B} & =\left(I_{1}-I_{2}\left(I_{1}-3 I_{2}\right)-0.53\right) V_{i j}^{1} \\
& -\left(I_{1}-4 I_{2}+4.476\right) V_{i j}^{2} \\
& +\left(16 I_{1}+4 I_{2}+3.748\right) V_{i j}^{3} \\
a_{i j}^{J} & =\left(I_{1}-I_{2}-0.71\right) V_{i j}^{1} \\
& +\left(I_{1}\left(I_{1}+I_{2}\right)\left(2 I_{1}-I_{2}\right)-0.71 I_{1}+0.5041\right) V_{i j}^{2} \\
& +\left(I_{1}\left(I_{2}-2\right)\left(I_{1}-I_{2}+0.73\right)-4 I_{1}+2 I_{2}+4.71\right) V_{i j}^{3} .
\end{aligned}
$$

A macroscopic inspection of the obtained models reveals a couple of key points:

1. The equations show that the models developed are highly non-linear with all tensor basis functions present, when compared with the Boussinesq approximation i.e. $a_{i j}^{\text {mod }}=$ $-V_{i j}^{1}$.

2. Comparing the constant coefficient of the first tensor basis function $\left(V_{i j}^{1}\right)$ from all the new models with the Boussinesq 

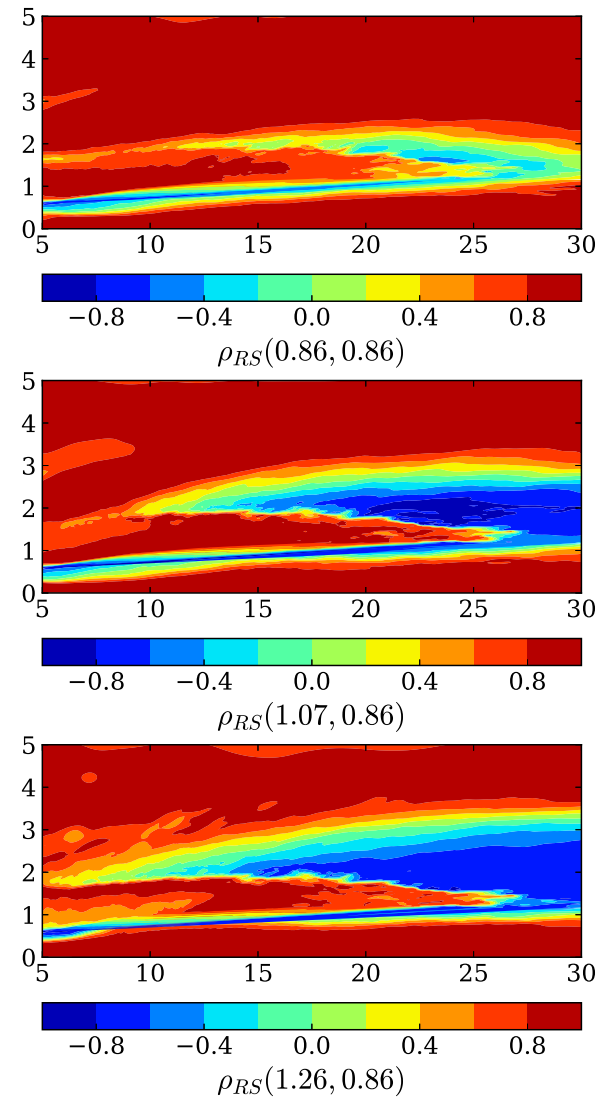
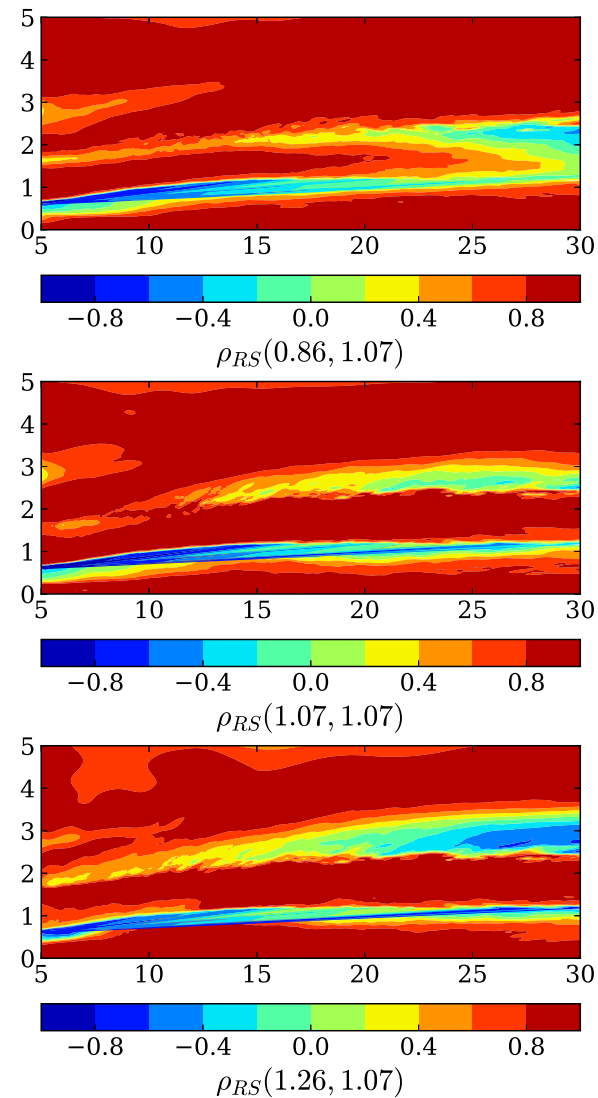
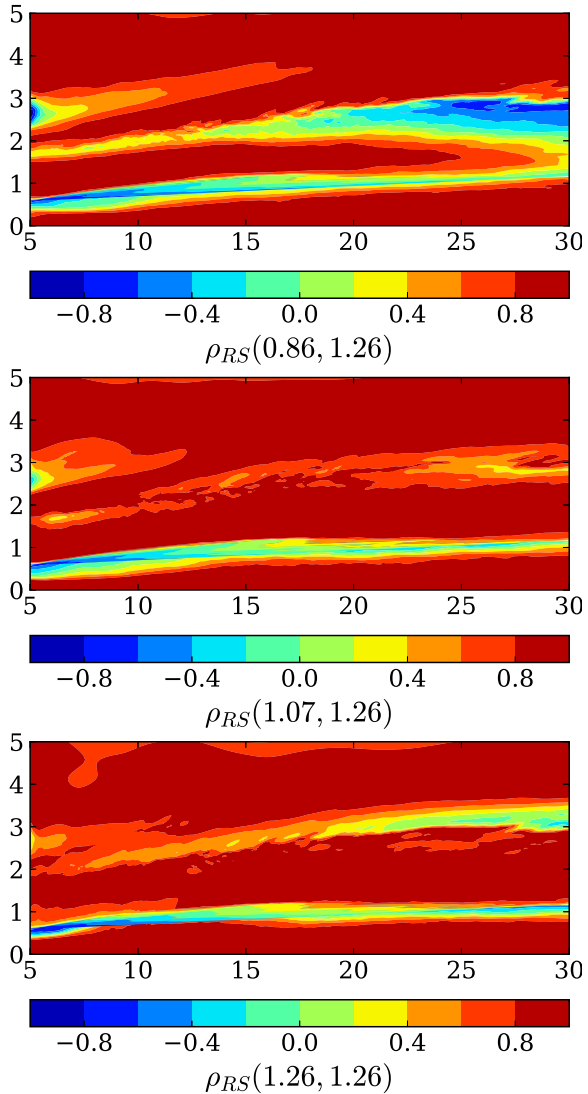

FIGURE 11. Two model closure generalisability.

approximation shows that the magnitudes are smaller. As an example, the $V_{i j}^{1}$ coefficients of $a_{i j}^{B}$ and $a_{i j}^{J}$ for MFR = 1.07 are $-0.30 \&-0.99$, respectively, compared with -1.0 for a linear model. This coefficient is an indicator of the amount of turbulence diffusion from the model. Thus, the developed closures recommend a lower amount of turbulence diffusion compared to the Boussinesq closure.

3. For each MFR, the boundary layer model has a much lower turbulence diffusion contribution compared with the jet/wake model, i.e. $-0.619 \&-1$ for MFR $=0.86$ and -0.53 $\&-0.71$ for MFR $=1.26$. Following the observations in Lav et al. [16] on the impact of this value in a URANS implementation, there will be a higher proportion of length scales being resolved in the boundary layer region since the turbulence closure adds a smaller amount of turbulence diffusion.

4. Even though the coefficients of $V_{i j}^{1}$ in the jet/wake region are comparable to the Boussinesq closure, it does not mean the Boussinesq closure is valid (see the closure's alignment in Fig. 6), rather, the model mechanism of just turbulence diffusion is not sufficient to capture the jet/wake dynamics and there is a need for other terms, which is evident from the other scalar invariant coefficients of $V_{i j}^{1}$ and tensor basis functions $V_{i j}^{2}, V_{i j}^{3}$.

Next, the $J, B$ models were stitched together and the alignments were computed, shown in Fig. 11 for all three MFRs. The label per sub-figure indicates the alignment with different reference and model anisotropies. As an example, $\rho_{R S}(1.26,0.86)$ refers to the alignment using the reference anisotropy for MFR $=1.26$ and the model anisotropy for MFR $=0.86$. The resulting $3 \times 3$ matrix of sub-figures thus is a test of generalisability of the developed models across other MFRs.

The contours show that the developed models show a much better alignment with the reference compared with the results obtained from the other training regions. Negative alignment values are accentuated away from the wall for $\rho_{R S}(1.07,0.86)$ and $\rho_{R S}(1.26,0.86)$, which correspond to the MFR $=0.86$ model applied to MFR $=1.07,1.26$ cases. This deviation is understandable due to the difference in the physics, as MFR $=0.86$ corresponds to a wall-wake flow while the other two MFRs correspond to wall-jets. It would also explain why the models developed on MFR $=1.07$ and 1.26 suffer in the same region for the MFR = 0.86 case $\left(\rho_{R S}(0.86,1.07)\right.$ and $\left.\rho_{R S}(0.86,1.26)\right)$. With the aim to find a model that works across different MFRs, a simple visual inspection indicates the two-zone model closure developed on MFR $=1.26$ provides the best overall fit in the alignment compared with the other two closures.

Thus, to assess the improvements with obtained closure, estimates of the modelled Reynolds stress profiles are compared with the reference dataset. Figure 12 shows the estimated stochastic Reynolds stresses for MFR $=1.26$ using the developed closure for MFR $=1.26$. The figure shows that the estimated result from the chosen closure matches the actual Reynolds stress extremely well, particularly in the near wall region, in terms of the shape and magnitude of the stresses. The estimated result from the Boussinesq approximation has also been added to the plots to demonstrate the extent of misprediction in the near wall region. Two clarifications about this figure are in order:

1. Since the training has been performed using just the stochastic anisotropy, the closure can provide an estimate of the stochastic contribution only, which is why the plots in 

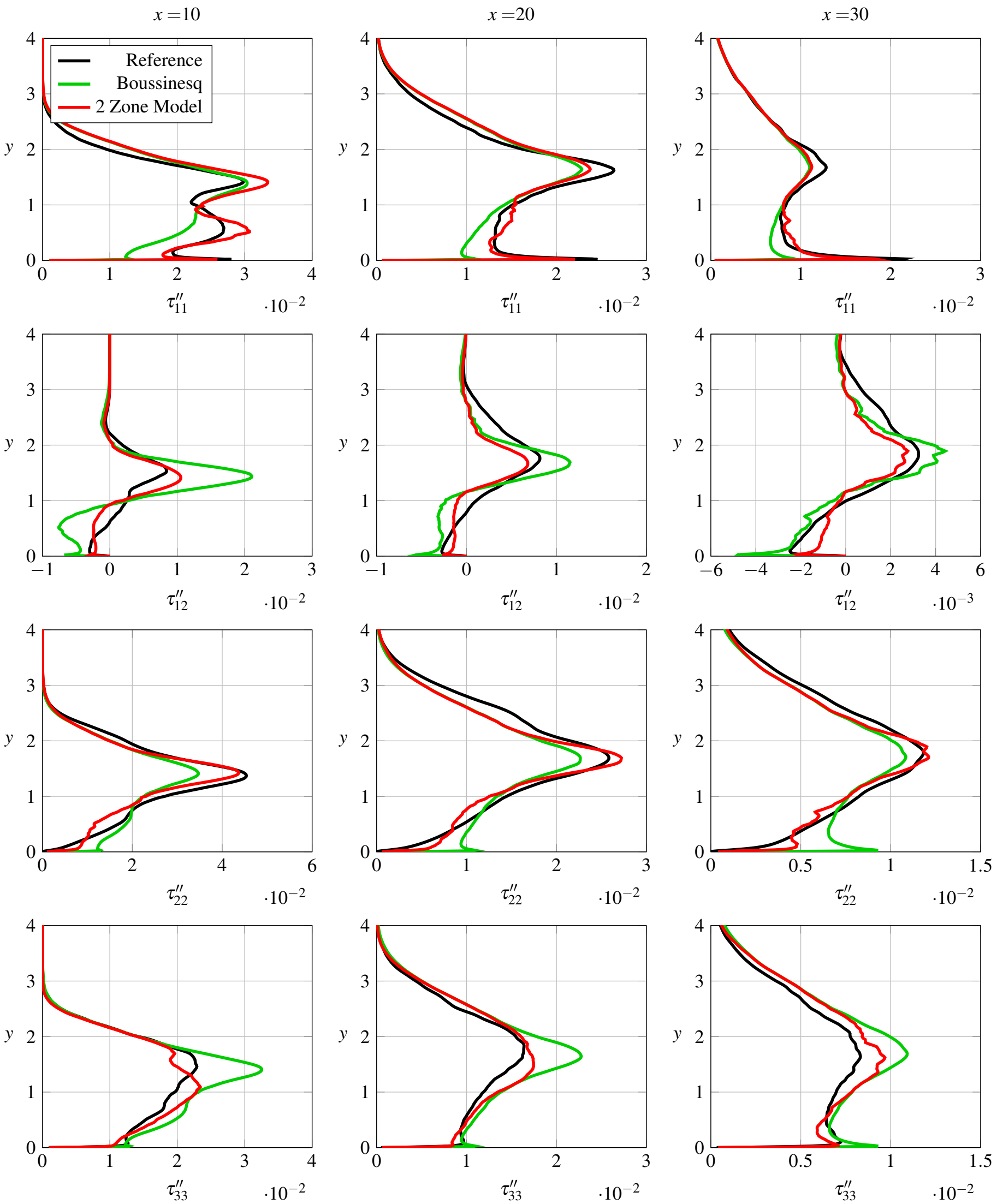

FIGURE 12. Estimated stochastic Reynolds stress profiles comparing the zonal turbulence closure with the reference from LES for MFR $=1.26$. Also shown is the estimated result from the Boussinesq closure. 
Fig. 12 show the stochastic part of the Reynolds stress. Upon implementation of this closure in a URANS solver, the total Reynolds stress would comprise the modelled/stochastic contribution from the developed closure and the resolved/deterministic contribution.

2. The stochastic Reynolds stress components have been constructed from the closure model using

$$
\tau_{i j}^{\prime \prime}=2 k^{\prime \prime} a_{i j}^{m o d}+2 k^{\prime \prime} \frac{\delta_{i j}}{3} .
$$

$a_{i j}^{\text {mod }}$ is constructed by stitching $a_{i j}^{B}$ and $a_{i j}^{J}$ in Eqn. (15). The terms $k^{\prime \prime}$ and the tensor basis and scalar invariant function values for $a_{i j}^{\text {mod }}$ are obtained from the reference dataset. Since these values would differ from the reference when obtained from URANS, a better way to highlight the difference between the obtained closure and the Boussinesq approximation is to look directly at the estimated anisotropy values, shown in Fig. 13. Anisotropy from the Boussinesq approximation for the normal components $\left(a_{i i}^{\prime \prime}\right)$ is close to zero, suggesting an isotropic distribution of the stochastic anisotropy. However, the reference shows that is not the case. The developed 2-zone closure on the other hand manages to capture the trends of the normal components, which should translate into actual improvement upon implementation.

These results represent the a priori analysis of the proposed framework and as such constitutes of only the first part of the complete framework. The second part, containing the a posteriori analysis, where the obtained models are implemented in URANS and calculations performed, will be presented in another study. This choice has been made to provide a more elaborate understanding of the developed turbulence closures, rooted in the fundamental physics of the flow.

\section{CONCLUSION}

In this study, the a priori part of a new framework was presented to improve prediction of the momentum mixing of wall-jet and wall-wake flows. The improvements are obtained by developing a non-linear turbulence closure using a machine-learning algorithm considering only the stochastic length scales. The geometry considered exhibits vortex shedding, which corresponds to the deterministic scales in the flow. Since these scales are coherent in nature, they do not represent turbulence, so turbulence closure development without these scales was shown to generate better models than closure development using all scales. An investigation was also conducted into the effect of choosing different spatial extents on the developed models with the observation that one closure was not enough to describe the full flow physics. Thus, a zonal turbulence closure approach was adopted, whereby two closures were obtained, one to describe the wall boundary layer and one to describe the jet/wake, away from the wall. The separation of these two regions was obtained through a flow based reasoning, using the streamwise velocity gradient in the wall normal direction, rather than a geometric consideration. Finally, a test of generalisability was extended to the developed closures by applying a closure from one mass flow ratio to the other mass flow ratios. It was observed that the model for the ratio of 1.26 performed best across the other ratios, including itself while, training on the ratio of 0.86 had the opposite effect,
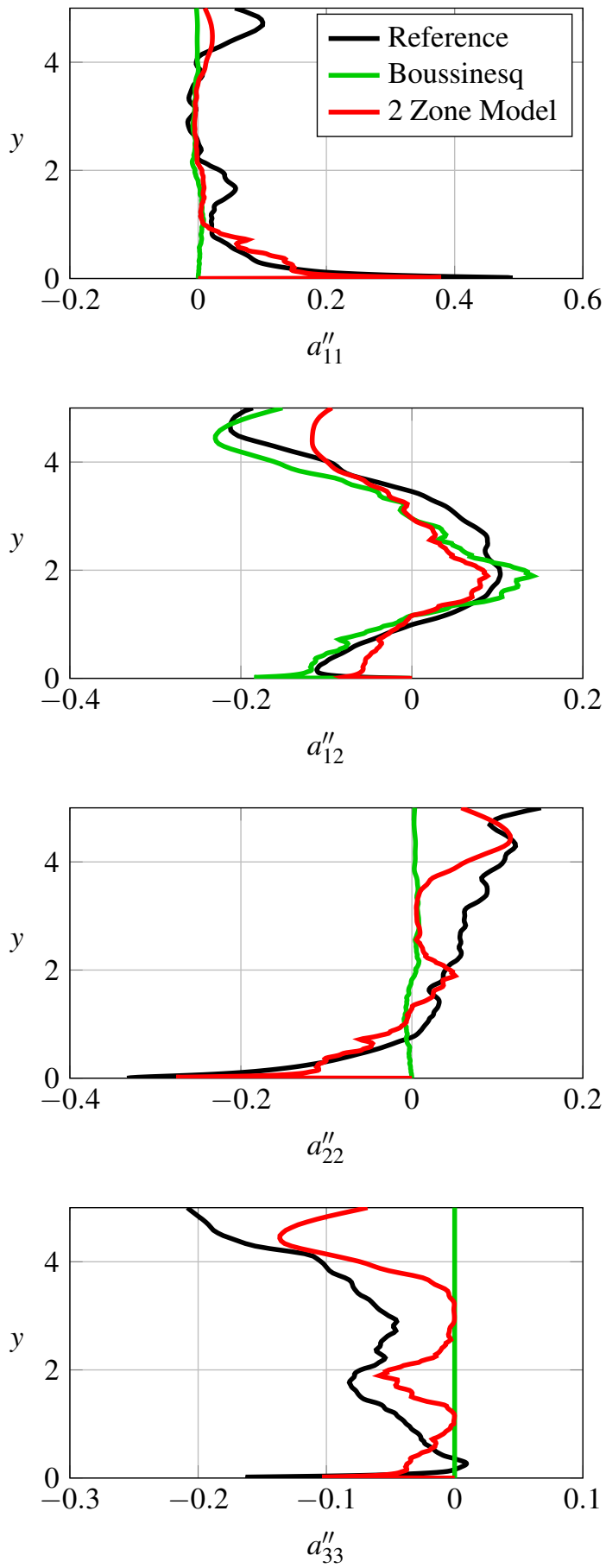

FIGURE 13. Estimated stochastic anisotropy profiles comparing the zonal turbulence closure with the reference from LES for MFR $=1.26$ at $x=30.0$. Also shown is the estimated result from the Boussinesq closure.

particularly in the region governed by the jet/wake. This was due to the fact that the 0.86 case was effectively a wall-wake while the other two ratios were wall-jets.

\section{ACKNOWLEDGMENT}

This work was supported by resources provided by the Pawsey Supercomputing Centre with funding from the Australian Government and the Government of Western Australia. The authors also acknowledge financial support from the Australian Research Council. 


\section{REFERENCES}

[1] Kacker, S. C., and Whitelaw, J. H., 1969. "An experimental investigation of the influence of slot-lip-thickness on the impervious-wall effectiveness of the uniform-density, twodimensional wall jet”. Int. J. Heat Mass Transf., 12(9), pp. 1196-1201.

[2] Kacker, S. C., and Whitelaw, J. H., 1971. "The turbulence characteristics of two-dimensional wall-jet and wall-wake flows". J. Appl. Mech., 38(1), pp. 239-252.

[3] Sandberg, R. D., Tan, R., Weatheritt, J., Ooi, A., Haghiri, A., Michelassi, V., and Laskowski, G., 2018. "Applying Machine Learnt Explicit Algebraic Stress and Scalar Flux Models To a Fundamental Trailing Edge Slot”. J. Turbomach., 140(October), pp. 1-13.

[4] Medic, G., and Durbin, P. a., 2005. "Unsteady Effects on Trailing Edge Cooling". J. Heat Transfer, 127(4), pp. 388-392.

[5] Joo, J., and Durbin, P., 2009. "Simulation of Turbine Blade Trailing Edge Cooling”. J. Fluids Eng., 131(2), p. 021102.

[6] Ivanova, E., and Laskowski, G. M., 2014. "LES and Hybrid RANS/LES of a Fundamental Trailing Edge Slot". In Turbomach. Tech. Conf. Expo. GT2014-25906, pp. 1-9.

[7] Kacker, S. C., and Whitelaw, J. H., 1968. "Some properties of the two-dimensional, turbulent wall jet in a moving stream". J. Appl. Mech., 35, pp. 641-651.

[8] Wallin, S., and Johansson, A. V., 2000. "An explicit algebraic Reynolds stress model for incompressible and compressible turbulent flows". J. Fluid Mech., 403, pp. 89-132.

[9] Duraisamy, K., Iaccarino, G., and Xiao, H., 2018. "Turbulence Modeling in the Age of Data". Annu. Rev. Fluid Mech.(April), pp. 1-23.

[10] Weatheritt, J., and Sandberg, R., 2016. "A novel evolutionary algorithm applied to algebraic modifications of the RANS stress-strain relationship". J. Comput. Phys., 325, pp. 22-37.

[11] Weatheritt, J., Sandberg, R. D., Ling, J., Saez, G., and Bodart, J., 2017. "A Comparative Study of Contrasting Machine Learning Frameworks Applied to RANS Modeling of Jets in Crossflow". In Turbomach. Tech. Conf. Expo. GT2017-63403, pp. 1-12.

[12] Weatheritt, J., Sandberg, R. D., Laskowski, G., and Michelassi, V., 2017. "Machine Learning For Turbulence Model Development Using A High-Fidelity HPT Cascase Simulation". In Turbomach. Tech. Conf. Expo. GT201763497, pp. 1-12.

[13] Menter, F. R., Kuntz, M., and Langtry, R., 2003. "Ten years of industrial experience with the SST turbulence model". Turbul. Heat Mass Transf., 4(1), pp. 625-632.

[14] Pope, S. B., 1975. "A more general effective-viscosity hypothesis". J. Fluid Mech., 72(2), pp. 331-340.

[15] Schmitt, F. G., 2007. “About Boussinesq's turbulent viscosity hypothesis: historical remarks and a direct evaluation of its validity". Comptes Rendus - Mec., 335(9-10), pp. 617-627.

[16] Lav, C., Sandberg, R. D., and Philip, J., 2018. "Improvement in Unsteady Wake Prediction Through Machine Learning based RANS Model Training". In Int. Symp. Unsteady Aerodyn. Aeroacoustics Aeroelasticity Turbomachines 15, no. September 24-27, ISUAAAT Scientific Committee, pp. 1-11.

[17] Hussain, A. K. M. F., and Reynolds, W. C., 1970. “The mechanics of an organized wave in turbulent shear flow". J. Fluid Mech., 41(02), pp. 241-258.

[18] Nicoud, F., and Ducros, F., 1999. "Subgrid-Scale Stress Modelling Based on the Square of the Velocity Gradient Tensor". Flow, Turbul. Combust., 62, pp. 183-200.

[19] Sandberg, R. D., Michelassi, V., Pichler, R., Chen, L., and Johnstone, R., 2015. "Compressible direct numerical simulation of low-pressure turbines: part I - methodology”. $J$. Turbomach., 137(5), pp. 051011-1-10.

[20] Jeong, J., and Hussain, F., 1995. "On the identification of a vortex". J. Fluid Mech., 285, pp. 69-94. 


\section{University Library}

\section{- M M N E R VA A gateway to Melbourne's research publications}

Minerva Access is the Institutional Repository of The University of Melbourne

Author/s:

Lav, C;Philip, J;Sandberg, R

Title:

A New Data-Driven Turbulence Model Framework for Unsteady Flows Applied to Wall-Jet and Wall-Wake Flows

Date:

2019-11-05

\section{Citation:}

Lav, C., Philip, J. \& Sandberg, R. (2019). A New Data-Driven Turbulence Model Framework for Unsteady Flows Applied to Wall-Jet and Wall-Wake Flows. Proceedings of ASME Turbo Expo 2019, 2A-2019, The American Society of Mechanical Engineers. https:// doi.org/10.1115/GT2019-90179.

Persistent Link:

http://hdl.handle.net/11343/241908 